\title{
Erratum: Time-Bin and Polarization Superdense Teleportation for Space Applications [Phys. Rev. Applied 14, 014044 (2020)]
}

\author{
Joseph C. Chapman $\odot,{ }^{1,2, *}$ Trent M. Graham, ${ }^{2,3}$ Christopher K. Zeitler, ${ }^{1,2}$ Herbert J. Bernstein, ${ }^{4}$ and \\ Paul G. Kwiat ${ }^{1,2}$ \\ ${ }^{1}$ Illinois Quantum Information Science and Technology Center, University of Illinois at Urbana-Champaign, \\ Urbana, Illinois 61801, USA \\ ${ }^{2}$ Department of Physics, University of Illinois at Urbana-Champaign, Urbana, Illinois 61801, USA \\ ${ }^{3}$ Department of Physics, University of Wisconsin-Madison, Madison, Wisconsin 53706, USA \\ ${ }^{4}$ Institute for Science \& Interdisciplinary Studies \& School of Natural Sciences, Hampshire College, Amherst, \\ Massachusetts 01002, USA
}

(Received 28 October 2020; published 12 November 2020)

DOI: 10.1103/PhysRevApplied.14.059902

Table I has been updated as seen below to correct several typographical errors in rows 13 and 21 where part of the last two entries in the row were switched. Furthermore, the states in the first four columns have been rearranged to make the phase convention of Appendix D consistent to allow for easier reproduction of the table. (Originally, we chose a phase convention which we thought made the table easier to reproduce by looking at Fig. 2.)

To make the phase convention consistent throughout Appendix D and correct an error during manuscript creation, $M$ should be right after the equals sign in Eqs. (D5)-(D8) as shown below.

$$
\begin{aligned}
& \text { Setang }_{1} \equiv M \times \mathrm{P}_{r} \times H_{1} \times Q_{1} \times \operatorname{Interf}_{P 1} \times Q_{2} \times H_{2} \times\left[\begin{array}{l}
1 \\
0
\end{array}\right] \\
& \text { Setang }_{2} \equiv M \times \mathrm{P}_{r} \times H_{1} \times Q_{1} \times \operatorname{Interf}_{P 1} \times Q_{2} \times H_{2} \times\left[\begin{array}{l}
0 \\
1
\end{array}\right], \\
& \text { Setang }_{3} \equiv M \times \mathrm{P}_{r} \times H_{1} \times Q_{1} \times \operatorname{Interf}_{P 2} \times Q_{3} \times H_{4} \times\left[\begin{array}{l}
1 \\
0
\end{array}\right], \quad \text { and } \\
& \text { Setang }_{4} \equiv M \times \mathrm{P}_{r} \times H_{1} \times Q_{1} \times \operatorname{Interf}_{P 2} \times Q_{3} \times H_{4} \times\left[\begin{array}{l}
0 \\
1
\end{array}\right]
\end{aligned}
$$

There was also a typographical error, where the second and third rows of Eq. (D10) were flipped. The correct version is

$$
\operatorname{Interf}_{P 2} \equiv\left[\begin{array}{ll}
0 & 0 \\
0 & 1 \\
1 & 0 \\
0 & 0
\end{array}\right]
$$

We thank Dr. Jason Gallicchio and his group for their help in catching the above typographical errors and encouraging us to make the tables in the manuscript reproducible with the given formulas and phase convention.

Additionally, the authors noticed a small error in Fig. 12. In the original publication, the data in the top and bottom panel of the right-most graphs were mistakenly offset from one another by $0.7^{\circ}$.

None of the changes discussed in this erratum affect any of the results, calculations, or conclusions in this paper.

*jchapmn2@illinois.edu 


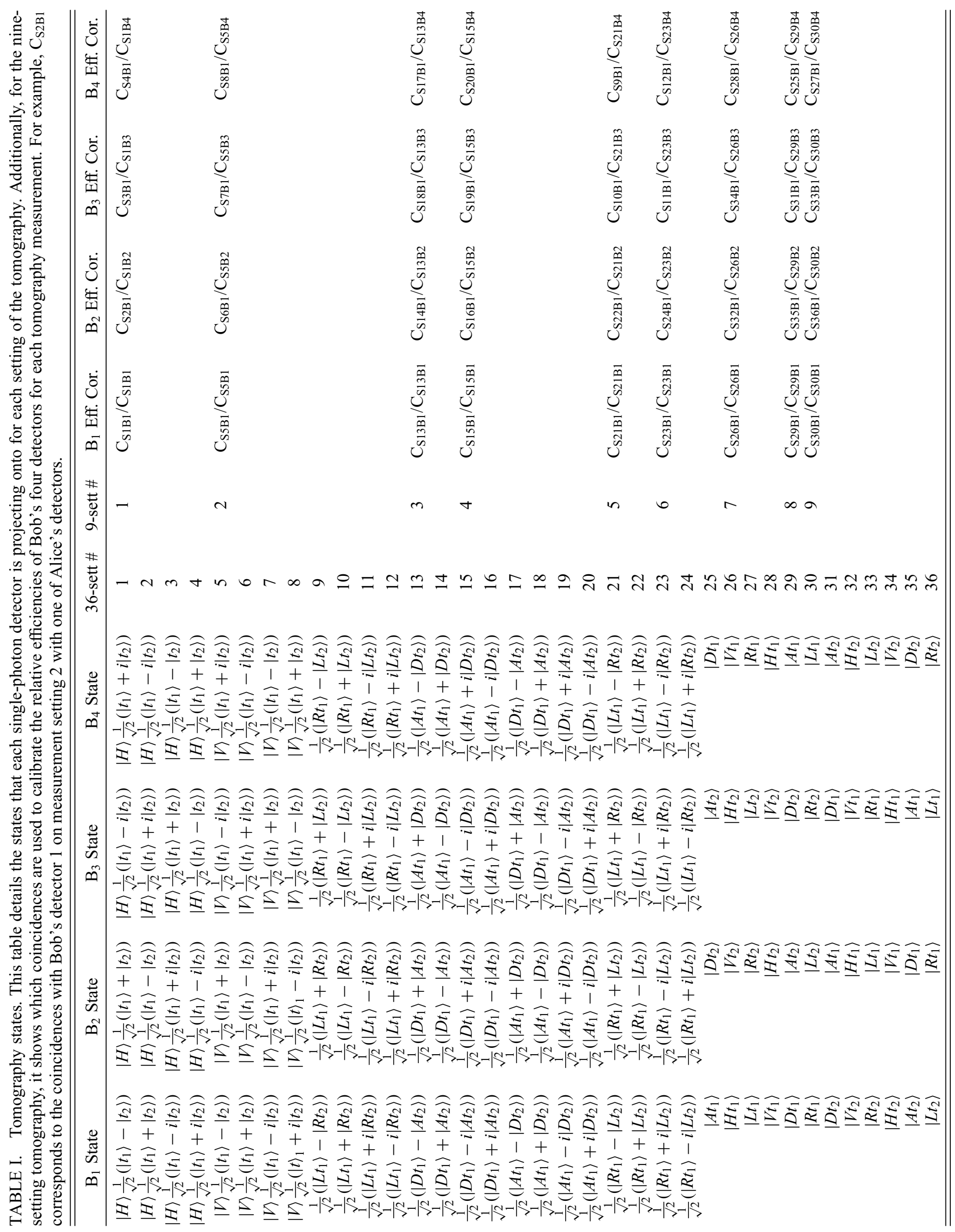


TABLE II. Tomography settings. This table details the angles of the wave plates and positions of the polarizers for each setting of the tomography. $\alpha_{1}, \alpha_{2}, \alpha_{3}$ are the angles of half-wave plates $1-3$; similarly $\beta_{1}, \beta_{2}, \beta_{3}$ are the angles of quarter-wave plates $1-3$. $T_{H}$ and $T_{V}$ are transmission settings of the removable polarizer.

\begin{tabular}{|c|c|c|c|c|c|c|c|c|c|}
\hline$\alpha_{1}$ & $\beta_{1}$ & $\alpha_{2}$ & $\beta_{2}$ & $\alpha_{3}$ & $\beta_{3}$ & $T_{H}$ & $T_{V}$ & 36 settings \# & 9 settings \# \\
\hline 22.5 & 90 & -22.5 & 0 & -22.5 & 135 & 1 & 0 & 1 & 1 \\
\hline 22.5 & 90 & 22.5 & 0 & 22.5 & 135 & 1 & 0 & 2 & \\
\hline 22.5 & 90 & 22.5 & 45 & -22.5 & 90 & 1 & 0 & 3 & \\
\hline 22.5 & 90 & -22.5 & 45 & 22.5 & 90 & 1 & 0 & 4 & \\
\hline 67.5 & 90 & -22.5 & 0 & -22.5 & 135 & 0 & 1 & 5 & 2 \\
\hline 67.5 & 90 & 22.5 & 0 & 22.5 & 135 & 0 & 1 & 6 & \\
\hline 67.5 & 90 & 22.5 & 45 & -22.5 & 90 & 0 & 1 & 7 & \\
\hline 67.5 & 90 & -22.5 & 45 & 22.5 & 90 & 0 & 1 & 8 & \\
\hline 0 & 45 & -22.5 & 0 & -22.5 & 90 & 1 & 1 & 9 & \\
\hline 0 & 45 & 22.5 & 0 & 22.5 & 90 & 1 & 1 & 10 & \\
\hline 0 & 45 & -22.5 & 45 & 22.5 & 135 & 1 & 1 & 11 & \\
\hline 0 & 45 & 22.5 & 45 & -22.5 & 135 & 1 & 1 & 12 & \\
\hline 22.5 & 90 & -22.5 & 0 & -22.5 & 90 & 1 & 1 & 13 & 3 \\
\hline 22.5 & 90 & 22.5 & 0 & 22.5 & 90 & 1 & 1 & 14 & \\
\hline 22.5 & 90 & 22.5 & 45 & -22.5 & 135 & 1 & 1 & 15 & 4 \\
\hline 22.5 & 90 & -22.5 & 45 & 22.5 & 135 & 1 & 1 & 16 & \\
\hline-22.5 & 90 & 22.5 & 0 & 22.5 & 90 & 1 & 1 & 17 & \\
\hline-22.5 & 90 & -22.5 & 0 & -22.5 & 90 & 1 & 1 & 18 & \\
\hline-22.5 & 90 & -22.5 & 45 & 22.5 & 135 & 1 & 1 & 19 & \\
\hline-22.5 & 90 & 22.5 & 45 & -22.5 & 135 & 1 & 1 & 20 & \\
\hline 0 & 135 & 22.5 & 0 & 22.5 & 90 & 1 & 1 & 21 & 5 \\
\hline 0 & 135 & -22.5 & 0 & -22.5 & 90 & 1 & 1 & 22 & \\
\hline 0 & 135 & 22.5 & 45 & -22.5 & 135 & 1 & 1 & 23 & 6 \\
\hline 0 & 135 & -22.5 & 45 & 22.5 & 135 & 1 & 1 & 24 & \\
\hline-22.5 & 90 & 0 & 0 & 0 & 90 & 1 & 1 & 25 & \\
\hline 0 & 90 & 0 & 0 & 0 & 90 & 1 & 1 & 26 & 7 \\
\hline 0 & 45 & 0 & 0 & 0 & 90 & 1 & 1 & 27 & \\
\hline 45 & 90 & 0 & 0 & 0 & 90 & 1 & 1 & 28 & \\
\hline 22.5 & 90 & 0 & 0 & 0 & 90 & 1 & 1 & 29 & 8 \\
\hline 0 & 135 & 0 & 0 & 0 & 90 & 1 & 1 & 30 & 9 \\
\hline-22.5 & 90 & 45 & 0 & 45 & 90 & 1 & 1 & 31 & \\
\hline 0 & 90 & 45 & 0 & 45 & 90 & 1 & 1 & 32 & \\
\hline 0 & 45 & 45 & 0 & 45 & 90 & 1 & 1 & 33 & \\
\hline 45 & 90 & 45 & 0 & 45 & 90 & 1 & 1 & 34 & \\
\hline 22.5 & 90 & 45 & 0 & 45 & 90 & 1 & 1 & 35 & \\
\hline 0 & 135 & 45 & 0 & 45 & 90 & 1 & 1 & 36 & \\
\hline
\end{tabular}



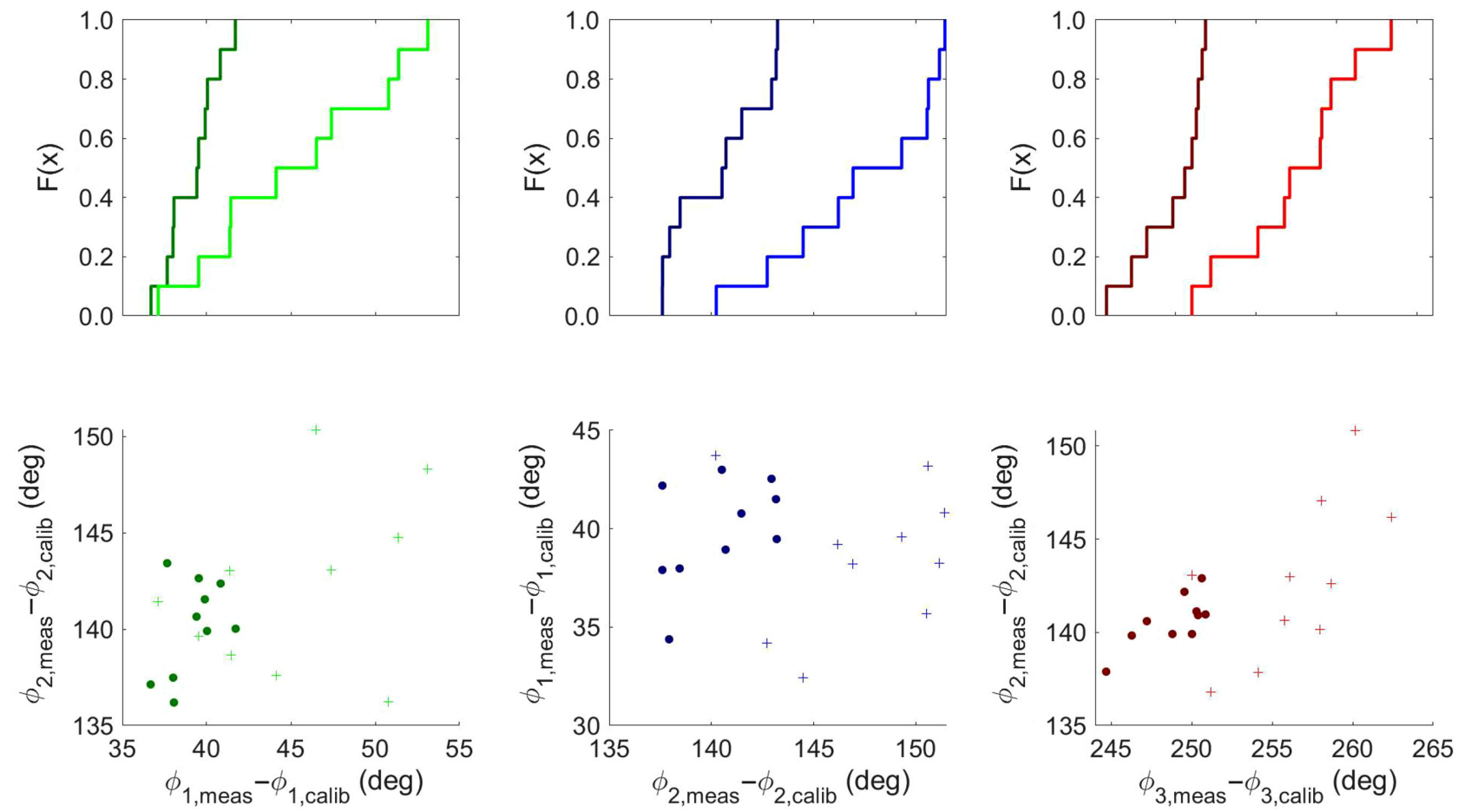

FIG. 12. Two-sample KS tests of very closely spaced distributions in phase space. (Top row) Empirical CDF of phase space data along horizontal axis. (Bottom row) Measured points in phase space. Crosses (dots) are measurements of the (un)displaced distributions. The distributions of crosses (dots) are centered over $45^{\circ}\left(39^{\circ}\right), 147^{\circ}\left(140^{\circ}\right), 256^{\circ}\left(249^{\circ}\right)$ for $\phi_{1}, \phi_{2}$, and $\phi_{3}$, respectively. 